\title{
The Clinicopathological Significance of the CXCR2 Ligands, CXCL1, CXCL2, CXCL3, CXCL5, CXCL6, CXCL7, and CXCL8 in Gastric Cancer
}

\author{
YURIE YAMAMOTO ${ }^{1,2}$, KENJI KURODA $^{1,2,3}$, TOMOHIRO SERA ${ }^{1,2,3}$, ATSUSHI SUGIMOTO $^{1,2,3}$, \\ SHUHEI KUSHIYAMA ${ }^{1,2,3}$, SADAAKI NISHIMURA ${ }^{1,2,3}$, SHINGO TOGANO ${ }^{1,2,3}$, TOMOHISA OKUNO $^{1,2,3}$, \\ MAMI YOSHII ${ }^{3}$, TATSURO TAMURA ${ }^{3}$, TAKAHIRO TOYOKAWA ${ }^{3}$, HIROAKI TANAKA ${ }^{3}$, \\ KAZUYA MUGURUMA ${ }^{3}$, MASAICHI OHIRA ${ }^{3}$ and MASAKAZU YASHIRO ${ }^{1,2,3}$ \\ ${ }^{1}$ Molecular Oncology and Therapeutics, Osaka City University Graduate School of Medicine, Osaka, Japan; \\ ${ }^{2}$ Cancer Center for Translational Research, Osaka City University Graduate School of Medicine, Osaka, Japan; \\ ${ }^{3}$ Department of Gastroenterological Surgery, Osaka City University Graduate School of Medicine, Osaka, Japan
}

\begin{abstract}
Background/Aim: We have previously reported that chemokine ( $C-X-C$ motif) receptor 2 (CXCR2) signaling was associated with the malignant progression of gastric cancer $(G C)$. We thus examined the clinicopathological significance of CXCR2 ligands, CXCL1, CXCL2, CXCL3, CXCL5, CXCL6, CXCL7, and CXCL8, in GC. Patients and Methods: The expression of CXCR2 ligands in 590 GC cases was investigated by immunohistochemistry. Results: The expression was as follows: CXCL1, 46.2\% (257/557); CXCL2, $20.7 \%$ (122/590); CXCL3, $17.1 \%$ (101/589); CXCL5/CXCL6, $2.9 \%$ (17/589); CXCL7, 36.4\% (215/590); and CXCL8 1.7\% (10/585) of the cases. High invasion depth was correlated with CXCL1 expression. Lymph node metastasis and peritoneal cytology positivity were correlated with high expression of CXCL1 and CXCL7. The prognoses of the CXCL1-positive patients were significantly poorer than those of the CXCL1-negative patients $(p<0.001)$. Conclusion: Among the CXCR2 ligands, CXCL7 and especially CXCL1, might play an important role in the malignant progression of GC via CXCR2 signaling.
\end{abstract}

Cancer progression has been recognized as not only the proliferation of tumor cells, but also the interaction between cancer cells and the surrounding stroma in the tumor

\footnotetext{
Correspondence to: Masakazu Yashiro, MD, Department of Surgical Oncology, Osaka City University Graduate School of Medicine, 1-43 Asahi-machi, Abeno-ku, Osaka 545-8585, Japan. Tel: +81 666453838, Fax: +81666466450, e-mail: m9312510@med.osakacu.ac.jp
}

Key Words: CXCR2 ligands, CXCL1, CXCL7, gastric cancer, tumor microenvironment. microenvironment (1). Among the tumor stromal cells, fibroblasts (especially cancer-associated fibroblasts) have been reported to be closely associated with tumor development in various solid carcinomas (2-4). We reported that chemokine (C-X-C motif) receptor 2 (CXCR2) signaling might play an important role in the pathogenic construction of tumor fibroblasts in the gastric tumor microenvironment, suggesting that gastric cancer (GC) cells might alter their adjacent stroma to form a permissive environment for tumor progression $(5,6)$. Following those findings, we reported that C-X-C ligand 1 (CXCL1) from GC cells stimulated the recruitment of bone marrow cells into the $\mathrm{GC}$ microenvironment via CXCR2 signaling (7). The reported CXCR2 ligands are not only CXCL1 but also CXCL2, CXCL3, CXCL5, CXCL6, CXCL7, and CXCL8. Until now, there has been only a single in vitro study on the significance of these CXCR2 ligands in GC (8).

\section{Patients and Methods}

Clinical materials. This study retrospectively analysed 590 GC patients who received gastrectomy at Osaka City University Hospital. GC tissues were obtained from each patient. The pathological diagnoses and classifications were made according to the Japanese classification of gastric carcinoma (15th edition). This study was approved by the Osaka City University Ethics Committee (approval number: 924). Written informed consent for research was obtained from all patients.

Immunohistochemical determination of CXCL1, CXCL2, CXCL3, CXCL5, CXCL6, CXCL7, and CXCL8. The immunohistochemical determination of CXCL1, CXCL2, CXCL3, CXCL5, CXCL6, CXCL7, and CXCL8 expression in gastric tumors was performed, as follows: We performed deparaffinization and slides were heated. After blocking endogenous peroxidase activity, the samples were incubated with anti-CXCL1 antibody (1:100; Abcam, Cambridge, 
UK), anti-CXCL2 antibody (1:200; Cloud-Clone Co., Katy, TX, USA), anti-CXCL3 antibody (1:200; Cloud-Clone Co.), antiCXCL5/CXCL6 antibody (1:100; Santa Cruz, Dallas, TX, USA), anti-CXCL7 antibody (1:250; Santa Cruz), and anti-CXCL8 antibody (1:250; R\&D systems, Minneapolis, MN, USA) for $1 \mathrm{~h}$ at room temperature. The samples were then incubated with biotinylated secondary antibody, followed by treatment with streptavidin-peroxidase reagent, and counterstaining with Mayer's hematoxylin. The expression levels of CXCL families were analyzed by both intensity of staining and percentage of stained cancer cells at the invading tumor front. CXCL1, CXCL2, CXCL3, CXCL5/CXCL6, CXCL7, and CXCL8 expression levels were evaluated as follows: the intensity was given scores $0-3(0=$ no, $1=$ weak, $2=$ moderate, $3=$ intense), and the percentage of immunepositive cells was given scores 0-3 (CXCL1: 0=0\%-10\%, 1=11\%$50 \%, 2=51 \%-80 \%, 3=81 \%-100 \%$, CXCL2: $0=0 \%, 1=1 \%-20 \%$, $2=21 \%-50 \%, 3=51 \%-100 \%$, CXCL3: $0=0 \%, 1=1 \%-20 \%, 2=21 \%-$ $60 \%, 3=61 \%-100 \%$, CXCL5 $/ 6: 0=0 \%, 1=1 \%-10 \%, 2=11 \%-40 \%$, $3=41 \%-100 \%$, CXCL7: $0=0 \%-20 \%, 1=21 \%-50 \%, 2=51 \%-70 \%$, $3=71 \%-100 \%$, and CXCL8: $0=0 \%-20 \%, 1=21 \%-30 \%, 2=31 \%-50 \%$, $3=51 \%-100 \%$ ). The two scores multiplied to gain the final result of $0-9$. Expression was considered positive when scores were CXCL1 $\geq 3$, CXCL2 $\geq 3$, CXCL3 $\geq 3$, CXCL5/CXCL6 $\geq 6$, CXCL7 $\geq 6$, and CXCL6 $\geq 2$. Evaluation was made by two double-blinded independent observers who were unaware of clinical data and outcome. When a different evaluation between the two independent observers was found, the evaluation was rechecked and discussed.

Statistical analysis. The chi-square test of Fisher's exact was used to decide the significance of difference between covariates. Survival periods were calculated with the Kaplan-Meier method and analyzed by log-rank test to compare cumulative survival durations among patient groups. In addition, the Cox proportional hazards model was used to compute univariate hazards ratios for the study parameters. The SPSS software program (SPSS Japan, Tokyo, Japan) was used for the analyses. A $p$-value $<0.05$ indicated a statistically significant difference.

\section{Results}

Immunostaining of CXCL1, CXCL2, CXCL3, CXCL5/CXCL6, CXCL7, and CXCL8. Staining of the members of the CXCL family was mainly at the cytoplasm and partially at the nuclei of cancer cells (Figure 1). In the 590 gastric tumors, the rates of positive expression were as follows: CXCL1, 258 (46.2\%); CXCL2, 122 (20.7\%); CXCL3, 101 (17.1\%); CXCL5/6, 17 (2.9\%); CXCL7, 215 (36.4\%); and CXCL8, 10 (1.7\%).

The relationship between clinicopathological features and the expression of CXCL1, CXCL2, CXCL3, CXCL5/CXCL6, CXCL7, and CXCL8 in gastric cancer. The clinicopathological characteristics of all 590 cases of GC according to their CXCL expression are summarized in Table I. The CXCL1-positive tumor group was significantly correlated with high age $(p<0.001)$, high T-stage $(p<0.001)$, lymph node metastasis $(p<0.001)$, lymphatic invasion $(p<0.001)$, and venous invasion $(p<0.001)$. The expression of CXCL2 in cancer cells was significantly correlated with low T-stage $(p=0.013)$, no lymph node metastasis $(p=0.033)$, and no peritoneal cytology $(p=0.01)$. The expression of CXCL3 in the cancer cells was significantly correlated with low infiltration $(p=0.002)$. The expression of CXCL5/CXCL6 in the cancer cells was not significantly correlated with any clinicopathological features. The expression of CXCL7 in the cancer cells was significantly correlated with high age $(p<0.001)$, diffuse type $(p=0.001)$, lymph node metastasis $(p=0.026)$, lymphatic invasion $(p=0.005)$, venous invasion $(p=0.023)$, infiltration pattern $(p<0.001)$, and peritoneal cytology negativity $(p=0.034)$. The expression of CXCL8 in the cancer cells was not correlated with any clinicopathological features.

Survival. The 5-year overall survival rate of the patients in the CXCL1-positive expression group was significantly worse compared to that of the CXCL1-negative group $(p<0.001$, Figure 2A). CXCL1 expression did not predict the prognosis of the GC patients at any stage (data not shown). The KaplanMeier survival curve indicated no significant difference in the 5-year overall survival rate between the positive and negative patients in CXCL2, CXCL3, CXCL5/CXCL6, CXCL7, and CXCL8 expression (Figure 2B).

Univariate and multivariate analyses of overall survival after surgery. Table II provides the results of the univariate and multivariate analyses of the patients' overall survival. The univariate analysis revealed that poor survival was significantly correlated with CXCL1-positive expression $(p<0.001)$, macroscopic type $4(p<0.001)$, diffuse type $(p<0.001)$, high $\mathrm{T}$ invasion $(p<0.001)$, lymph node metastasis $(p<0.001)$, venous invasion $(p<0.001)$, infiltration type 'c' $(p<0.001)$, hepatic metastasis $(p<0.001)$, peritoneal metastasis $(p<0.001)$, other metastasis $(p<0.001)$, and age $\geq 70$ years $(p=0.002)$. The multivariate analysis revealed that the factors that were significantly correlated with poor survival were CXCL1-positive expression $(p=0.039)$, macroscopic type $4(p<0.001)$, high $\mathrm{T}$ invasion $(p=0.009)$, lymph node metastasis $(p<0.001)$, hepatic metastasis $(p=0.001)$, peritoneal metastasis $(p<0.001)$, other metastasis $(p<0.001)$, and age $\geq 70$ years $(p=0.006)$.

\section{Discussion}

We investigated the expression of the CXCR2 ligands CXCL1, CXCL2, CXCL3, CXCL5/CXCL6, CXCL7, and CXCL8 in 590 gastric tumors. Expression on cancer cells was frequent for CXCL1 (46.2\%), followed by CXCL7 (36.4\%), CXCL2 (20.7\%), and CXCL3 (17.1\%). In contrast, CXCL5/CXCL6 (2.9\%) and CXCL8 (1.7\%) expression on GC cells was rare. CXCL1 has been reported to be expressed in 36\%-56\% of GC cases (8-10), and our present observation of a $46.2 \%$ CXCL1 expression rate is similar to these reports. CXCL1 has been described as a prognostic factor in 


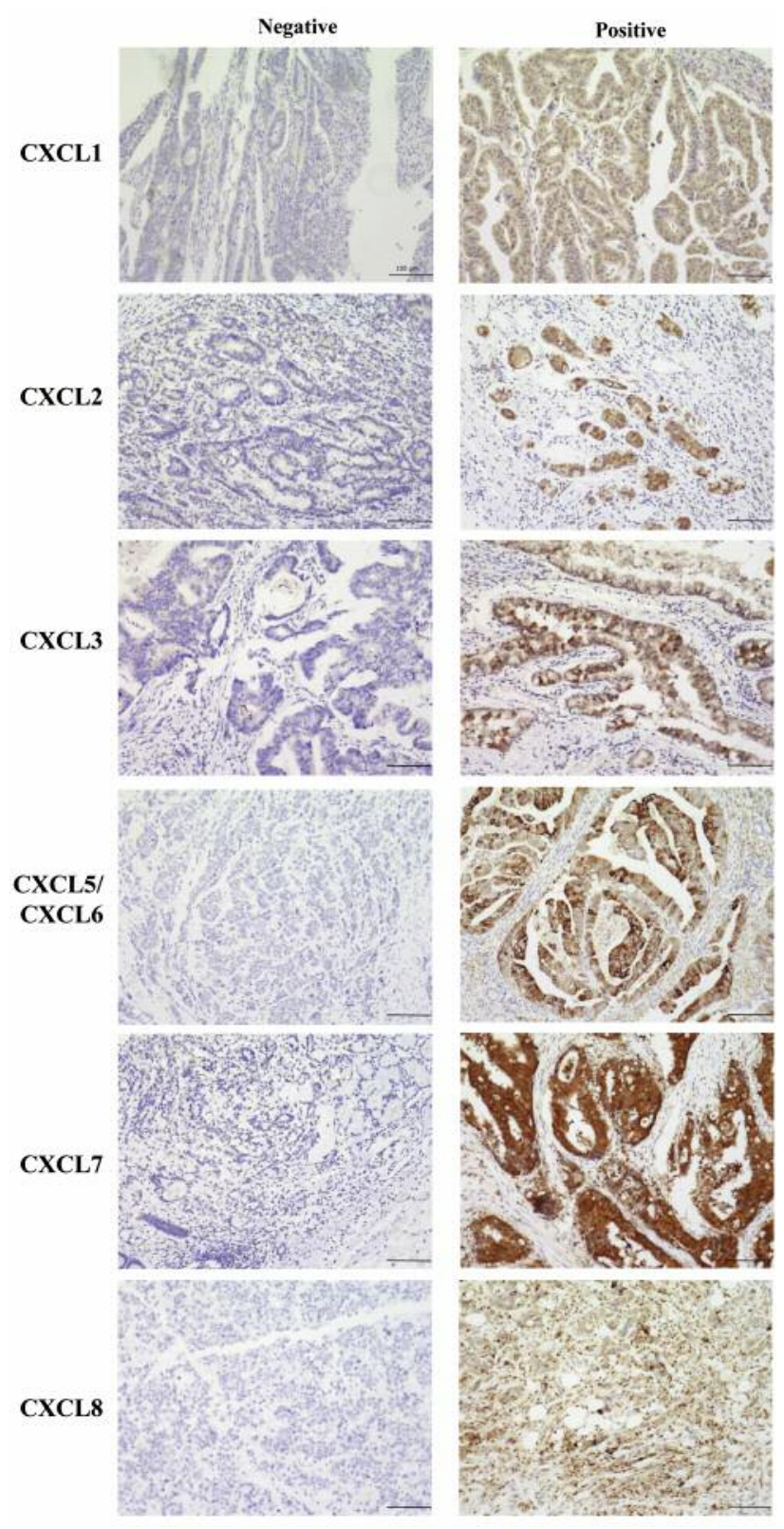

Figure 1. Representative images of CXCL1, CXCL2, CXCL3, CXCL5/6, $C X C L 7$, and $C X C L 8$ expression in gastric cancer. $C X C L 1, C X C L 2$, CXCL3, CXCL5/6, CXCL7 and CXCL8 were mainly expressed at the cytoplasm of cancer cells. $(\times 200)$. Bar: $100 \mu \mathrm{m}$.

various cancers (11) including hepatocellular carcinoma (HCC) (12), laryngeal squamous cell carcinoma (13), and colorectal cancer (14). In our present series of GC patients, CXCL1 was correlated with poor prognosis, and CXCL1 expression was correlated with features of the malignant potential of cancer, i.e., high T-stage, lymph node metastasis, lymphatic invasion, and venous invasion. These findings indicated that CXCL1 might play an important role in the progression of GC.

Kaplan-Meier survival curves indicated that the 5-year overall survival rate of the GC patients in the CXCL1positive group was significantly worse than that of the CXCL1-negative group. In addition, the multivariate analysis revealed that CXCL1 expression was an independent prognostic factor. These findings suggest that CXCL1 could be a useful prognostic factor for patients with GC.

The reported expression of CXCL7 in Wilms tumor $(\mathrm{n}=40)$ was $87.5 \%(15)$. CXCL7 expression was detected in $36.4 \%$ of the GC cases in the present investigation, and was correlated with the diffuse type, lymph node metastasis, lymphatic invasion, and venous invasion, although no significant difference in the 5-year survival rate was found between the CXCL7-positive and CXCL7-negative groups. CXCL7 might be partially associated with the malignant potential of GC cells.

CXCL2 expression in tumors has been reported only in HCC (16); there are no reports of CXCL2 expression in other cancers, including GC. The rate of CXCL2 expression in our series of 590 gastric cancer cases was $20.7 \%$. No significant difference in the 5-year survival rate was shown between the CXCL2-positive and CXCL2-negative groups, but the expression of CXCL2 was negatively correlated with T-stage, lymph node metastasis, and cytology. These findings indicate that CXCL2 might not play an important role in the progression of GC.

The rate of CXCL3 expression in cases of prostate cancer has been reported to be $21.7 \%$ (17). In the present study, the rate of CXCL3 expression in gastric cancer was $17.1 \%$. We observed that CXCL3 expression was associated with the infiltration pattern but with no other clinicopathological factors, including prognosis. Together these findings suggest that CXCL3 does not play an important role in the progression of GC.

Our results suggest that CXCL1, CXCL7, CXCL2, and CXCL3 produced from GC cells are involved in CXCR2 signaling. CXCL1 and CXCL7 and especially CXCL1 may have important functions in the progression of gastric cancer, but CXCL2 and CXCL3 expression may not.

CXCL5/CXCL6 expression was rare $(2.9 \%, \mathrm{n}=589)$ in this study. The CXCL5 expression in 155 cases of gastric cancer was reported to be $45.2 \%$ (8). CXCL6 expression has not been studied in any type of cancer. CXCL8 expression was also rare among our present patients $(1.7 \%$; $n=589)$, but a study of 22 patients with GC reported that CXCL8 expression was $100 \%$ (10). One of the possible reasons for these differences in the rates of CXCL5/CXCL6 and CXCL8 expression is based on the difference in the antibodies used in each study. The expression of CXCL5/6 and CXCL8 was not correlated with any of the clinicopathological prognostic factors in the present 


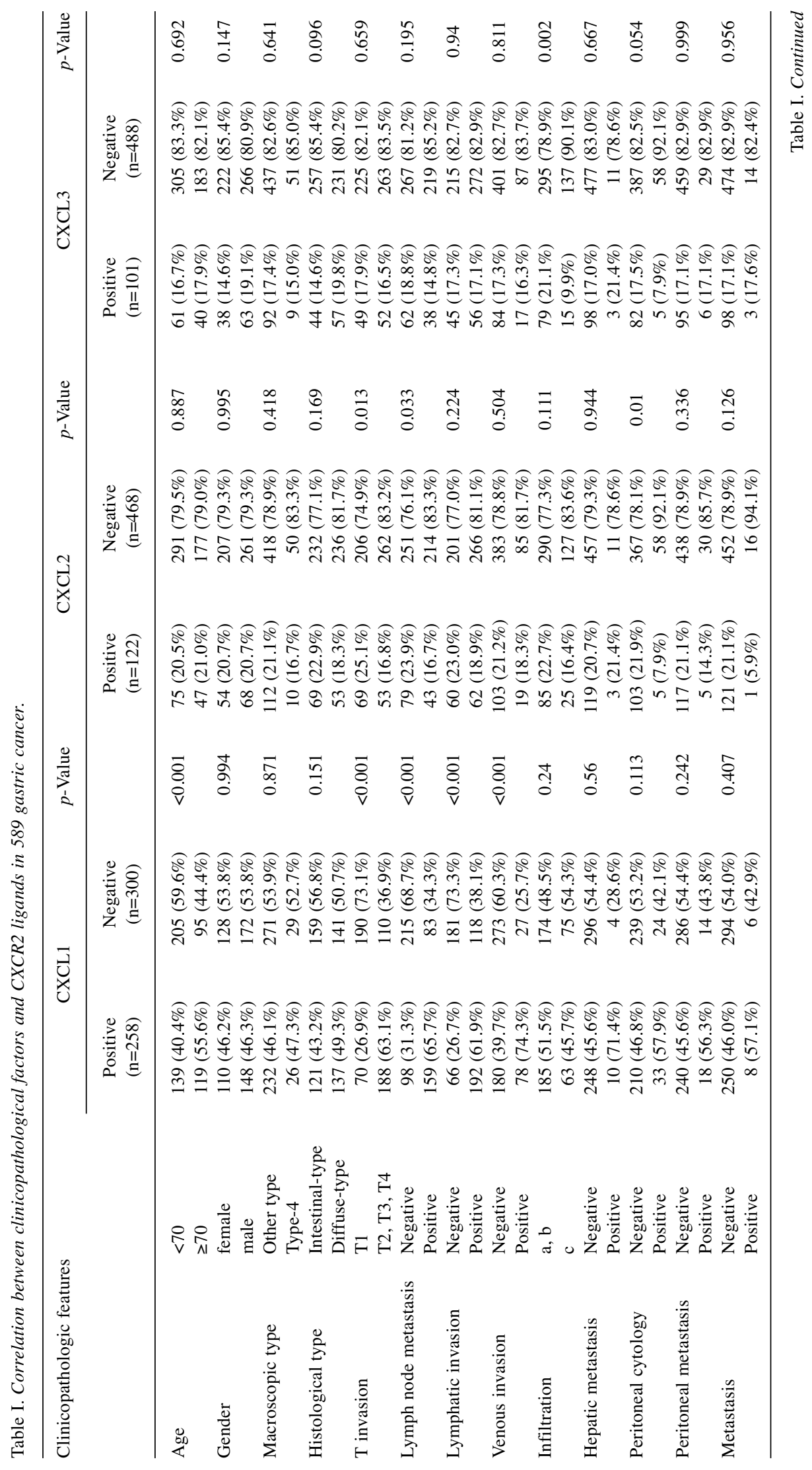




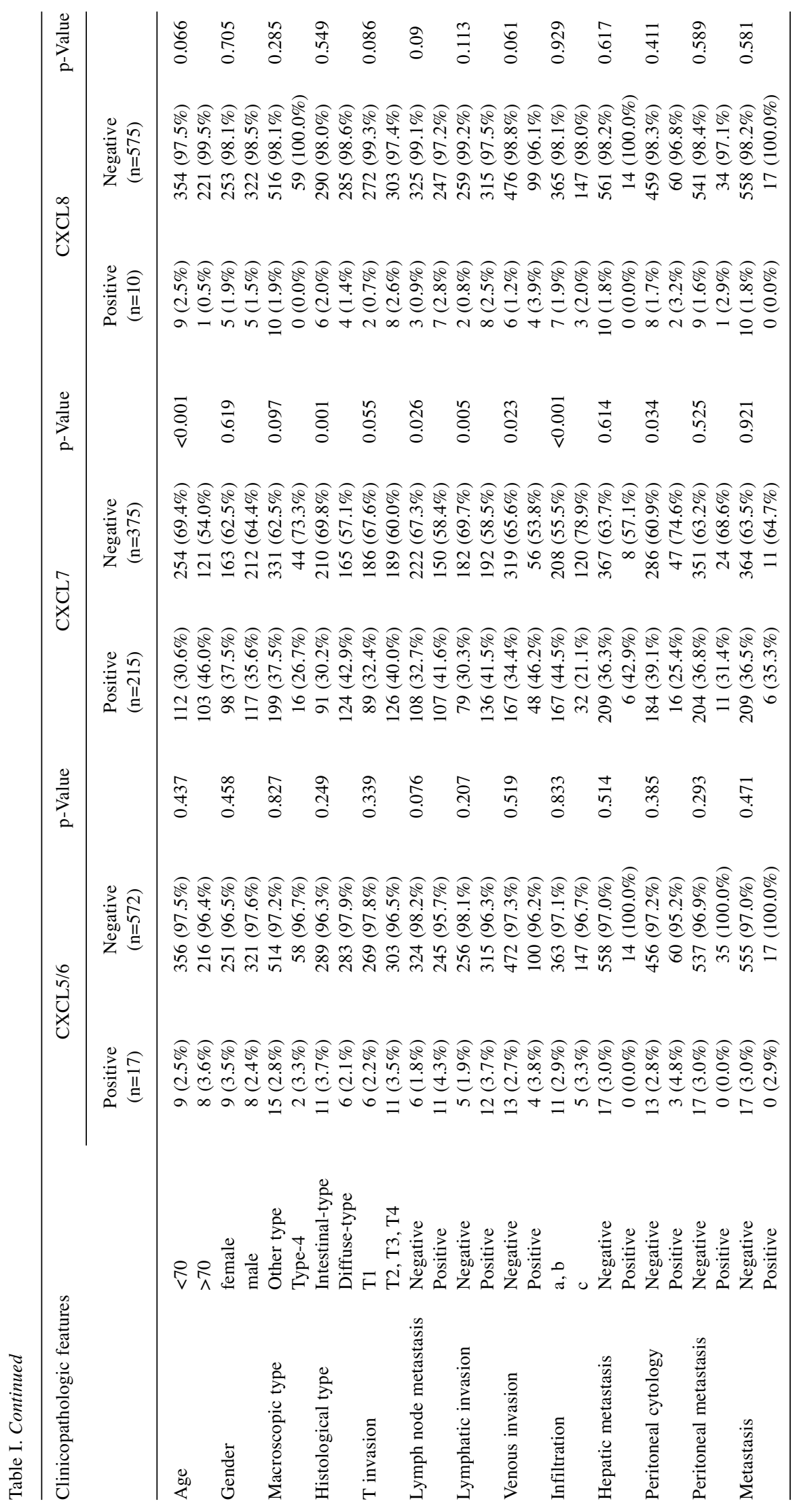




\section{A CXCL1}
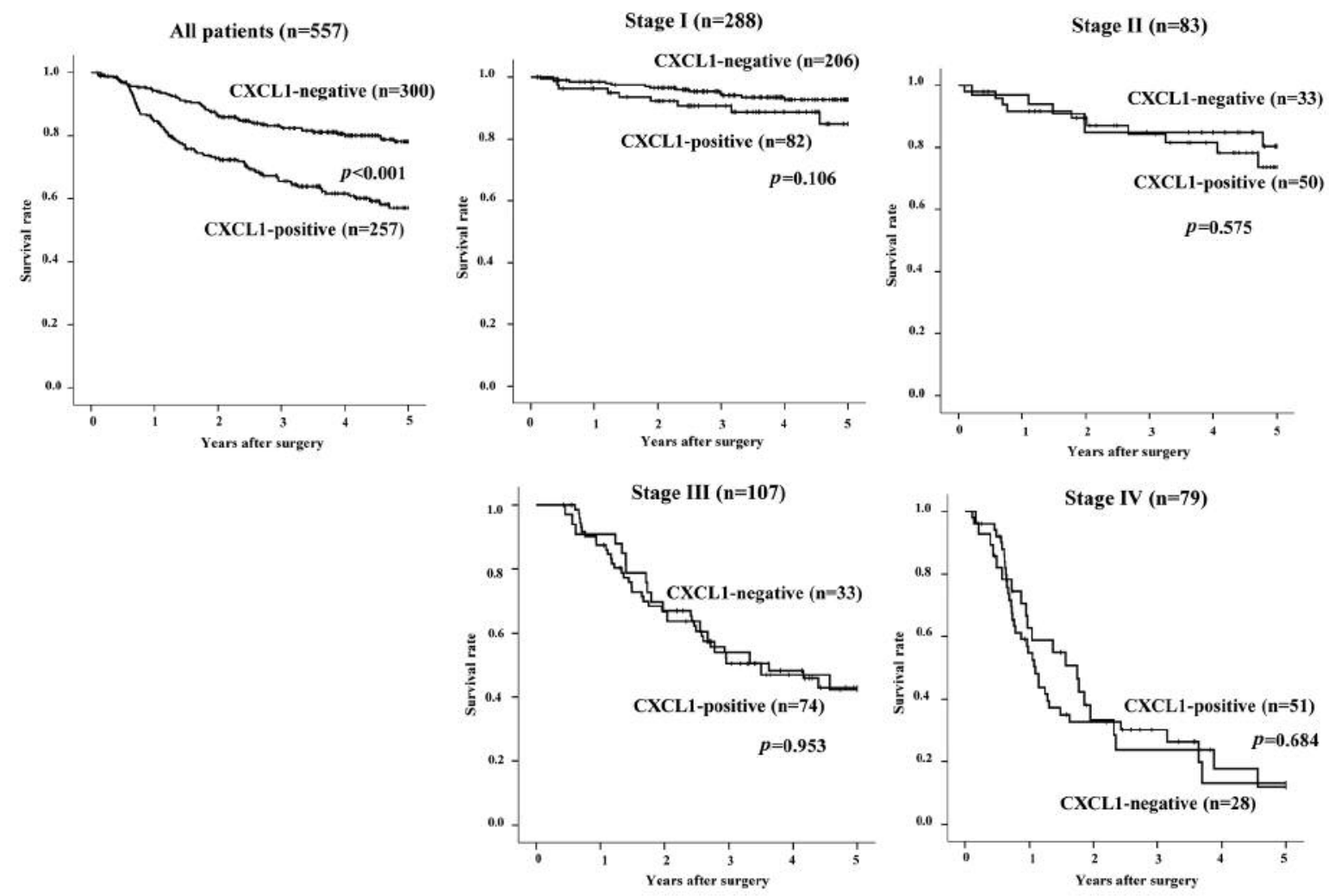

B

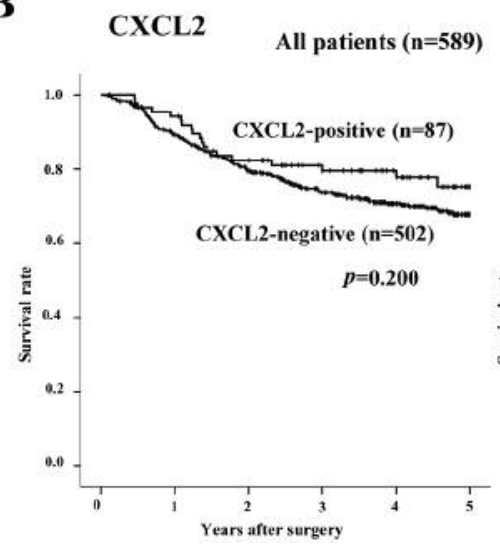

CXCL3

All patients $(\mathbf{n}=\mathbf{5 8 8})$

CXCL5/6

All patients $(\mathrm{n}=\mathbf{5 8 8})$
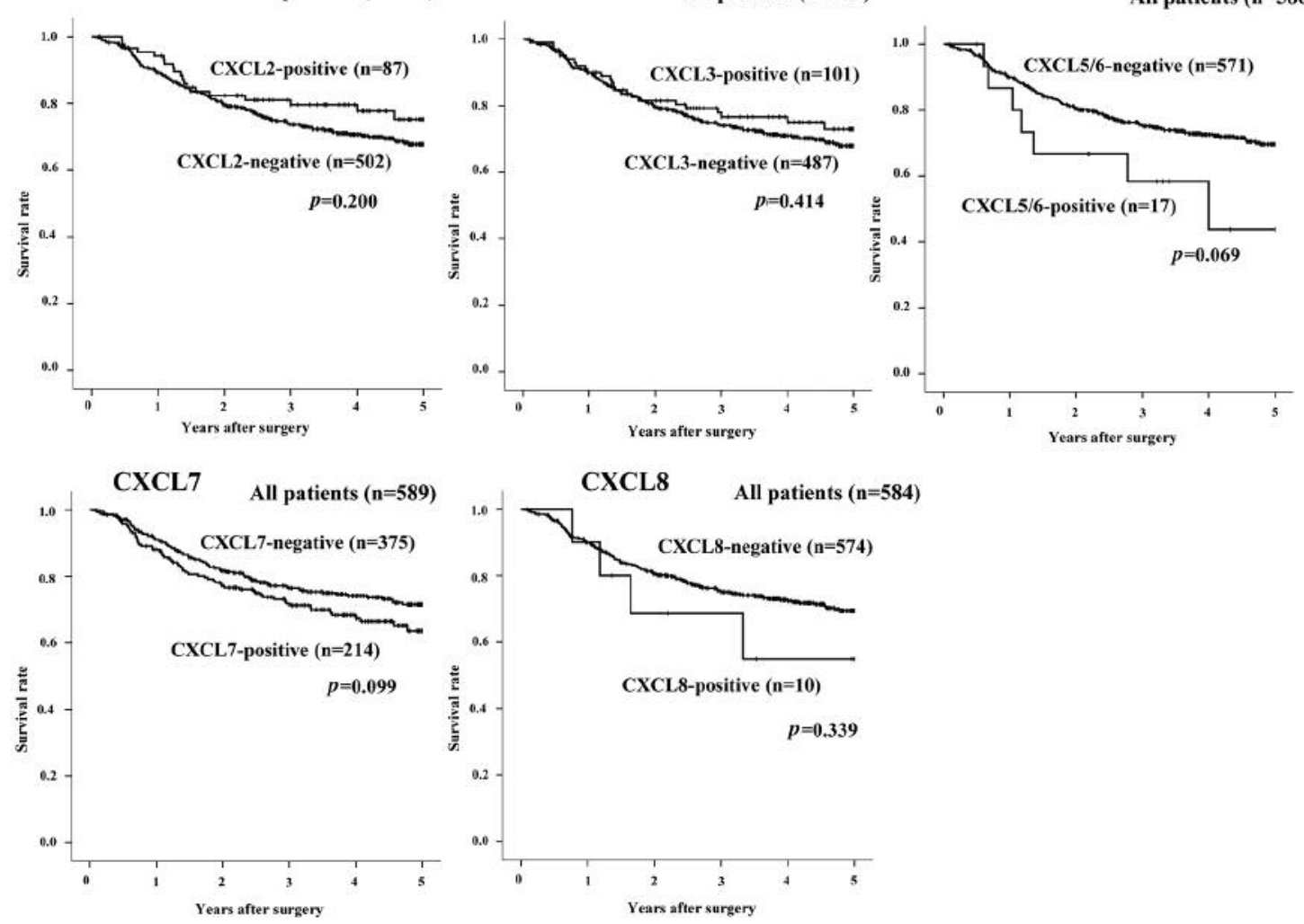
Table II. Univariate and multivariate Cox multiple regression analysis with respect to overall survival after surgery in 589 patients with gastric carcinoma.

\begin{tabular}{|c|c|c|c|c|c|c|}
\hline \multirow[b]{2}{*}{ Parameter } & \multicolumn{3}{|c|}{ Univariate } & \multicolumn{3}{|c|}{ Multivariate } \\
\hline & Hazard ratio & $95 \%$ CI & $p$-Value & Hazard ratio & $95 \% \mathrm{CI}$ & $p$-Value \\
\hline CXCL1 Positive & 2.272 & $1.632-3.161$ & $<0.001$ & 1.469 & $1.109-2.117$ & 0.039 \\
\hline CXCL2 Positive & 0.732 & $0.453-1.182$ & 0.202 & & & \\
\hline CXCL3 Positive & 0.835 & $0.54-1.289$ & 0.414 & & & \\
\hline CXCL5/6 Positive & 1.992 & $0.933-4.252$ & 0.075 & & & \\
\hline CXCL7 Positive & 1.305 & $0.950-1.792$ & 0.1 & & & \\
\hline CXCL8 Positive & 1.615 & $0.598-4.360$ & 0.344 & & & \\
\hline Macroscopic type: type-4 & 7.474 & $5.288-10.564$ & $<0.001$ & 2.619 & $1.669-4.109$ & $<0.001$ \\
\hline Histological type: diffuse-type & 0.556 & $0.403-0.766$ & $<0.001$ & 0.843 & $0.556-1.254$ & 0.399 \\
\hline T stage & 7.98 & $4.992-12.756$ & $<0.001$ & 2.27 & $1.233-4.182$ & 0.009 \\
\hline Lymph node metastasis: positive & 8.337 & $5.556-12.610$ & $<0.001$ & 3.229 & $1.909-5.464$ & $<0.001$ \\
\hline Venous invasion: positive & 3.291 & $2.379-4.554$ & $<0.001$ & 1.14 & $0.789-1.649$ & 0.485 \\
\hline Infiltration type c & 1.817 & $1.315-2.511$ & $<0.001$ & 1.008 & $0.668-1.520$ & 0.971 \\
\hline Hepatic metastasis: positive & 6.555 & $3.696-11.628$ & $<0.001$ & 3.088 & $1.599-5.964$ & 0.001 \\
\hline Peritoneal metastasis: positive & 9.11 & $6.016-13.796$ & $<0.001$ & 2.749 & $1.624-4.654$ & $<0.001$ \\
\hline Metastasis: Positive & 6.386 & $3.741-10.900$ & $<0.001$ & 3.523 & $1.864-6.659$ & $<0.001$ \\
\hline Age $\geq 70$ & 1.65 & $1.210-2.251$ & 0.002 & 1.636 & $1.152-2.324$ & 0.006 \\
\hline
\end{tabular}

study. These findings may indicate that CXCL5, CXCL6, and CXCL8 do not play important role in the CXCR2 signaling in the gastric cancer tumor microenvironment.

In conclusion, among the CXCR2 ligands, CXCL7 and especially CXCL1 were frequently expressed on gastric cancer cells. CXCL1 was closely associated with the malignant potential of this cancer, and it was an independent prognostic factor. CXCL1 might play an important role in the malignant progression of gastric cancer via CXCR2 signaling.

\section{Conflicts of Interest}

There are no financial or other interests with regard to the submitted manuscript that might be construed as a conflict of interest.

\section{Authors' Contributions}

Y.Y. performed the experiments of this study, interpreted the data and wrote the manuscript; Y.M., designed the experiments of this study, interpreted the data and edited the manuscript; K.K., T.S.,

Figure 2. Overall survival of gastric cancer patients based on CXCLs expression in gastric cancer cells. A: The Kaplan-Meier survival curves indicate that the overall survival of patients, at all disease stages, with CXCL1-positive expression was significantly worse than that of patients with CXCL1-negative expression $(p<0.001)$. B: In contrast, no significant difference in overall survival was found between patients with positive and negative expression of CXCL2, CXCL3, CXCL5,6, CXCL7, and CXCL8.
A.S., S.K., S.T., and T.O. contributed to the immunohistochemical analysis; M.Y., T.T., T.T., H.T., K.M. and S.N. collected the gastric cancer specimen; M.O. helped draft the manuscript.

\section{Acknowledgements}

This study is partially founded by KAKENHI Grant-in-Aid for Scientific Research, Nos. 18H02883 (M.Y.).

\section{References}

1 De Wever O and Mareel M: Role of tissue stroma in cancer cell invasion. J Pathol 200(4): 429-447, 2003. PMID: 12845611. DOI: $10.1002 /$ path.1398

2 Fuyuhiro Y, Yashiro M, Noda S, Kashiwagi S, Matsuoka J, Doi Y, Kato Y, Muguruma K, Sawada $\mathrm{T}$ and Hirakawa K: Myofibroblasts are associated with the progression of scirrhous gastric carcinoma. Exp Ther Med 1(4): 547-551, 2010. PMID: 22993574. DOI: 10.3892/etm_00000086

3 Kalluri R and Zeisberg M: Fibroblasts in cancer. Nat Rev Cancer 6(5): 392-401, 2006. PMID: 16572188. DOI: $10.1038 / \mathrm{nrc} 1877$

4 Yashiro $M$ and Hirakawa K: Cancer-stromal interactions in scirrhous gastric carcinoma. Cancer Microenviron 3(1): 127 135, 2010. PMID: 21209779. DOI: 10.1007/s12307-0100036-5

5 Kasashima H, Yashiro M, Nakamae H, Kitayama K, Masuda G, Kinoshita H, Fukuoka T, Hasegawa T, Nakane T, Hino M, Hirakawa $\mathrm{K}$ and Ohira $\mathrm{M}$ : Cxcl1-chemokine (c-x-c motif) receptor 2 signaling stimulates the recruitment of bone marrowderived mesenchymal cells into diffuse-type gastric cancer stroma. Am J Pathol 186(11): 3028-3039, 2016. PMID: 27742059. DOI: 10.1016/j.ajpath.2016.07.024

6 Yashiro M, Matsuoka $\mathrm{T}$ and Ohira M: The significance of scirrhous gastric cancer cell lines: The molecular characterization 
using cell lines and mouse models. Hum Cell 31(4): 271-281, 2018. PMID: 29876827. DOI: 10.1007/s13577-018-0211-4

7 Kasashima H, Yashiro M, Nakamae H, Masuda G, Kinoshita H, Morisaki T, Fukuoka T, Hasegawa T, Nakane T, Hino M, Hirakawa $\mathrm{K}$ and Ohira M: Clinicopathologic significance of the cxcl1-cxcr2 axis in the tumor microenvironment of gastric carcinoma. PLoS One 12(6): e0178635, 2017. PMID: 28575019. DOI: 10.1371 /journal.pone.0178635

8 Zhou Z, Xia G, Xiang Z, Liu M, Wei Z, Yan J, Chen W, Zhu J, Awasthi N, Sun X, Fung KM, He Y, Li M and Zhang C: A c-Xc chemokine receptor type 2-dominated cross-talk between tumor cells and macrophages drives gastric cancer metastasis. Clin Cancer Res 25(11): 3317-3328, 2019. PMID: 30796034. DOI: 10.1158/1078-0432.CCR-18-3567

9 Eck M, Schmausser B, Scheller K, Brandlein S and MullerHermelink HK: Pleiotropic effects of cxc chemokines in gastric carcinoma: Differences in cxcl8 and cxcl1 expression between diffuse and intestinal types of gastric carcinoma. Clin Exp Immunol 134(3): 508-515, 2003. PMID: 14632759. DOI: $10.1111 /$ j.1365-2249.2003.02305.x

10 Wei ZW, Xia GK, Wu Y, Chen W, Xiang Z, Schwarz RE, Brekken RA, Awasthi N, He YL and Zhang CH: Cxcl1 promotes tumor growth through vegf pathway activation and is associated with inferior survival in gastric cancer. Cancer Lett 359(2): 335343, 2015. PMID: 25641338. DOI: 10.1016/j.canlet.2015.01.033

11 Zhang Z, Chen Y, Jiang Y, Luo Y, Zhang H and Zhan Y: Prognostic and clinicopathological significance of cxcll in cancers: A systematic review and meta-analysis. Cancer Biol Ther: 1-9, 2019. PMID: 31387444. DOI: 10.1080/ 15384047.2019.1647056

12 Cao Z, Fu B, Deng B, Zeng Y, Wan X and Qu L: Overexpression of chemokine (c-X-c) ligand 1 (cxcl1) associated with tumor progression and poor prognosis in hepatocellular carcinoma. Cancer Cell Int 14(1): 86, 2014. PMID: 25298747. DOI: 10.1186/s12935-014-0086-8
13 Han L, Liu W, Chen Y, Wu H, Zhang Y and Jiang B: Groalpha expression and its prognostic implications in laryngeal squamous cell carcinoma. Neoplasma 62(1): 152-158, 2015. PMID: 25563380. DOI: 10.4149/neo_2015_020

14 Zhuo C, Wu X, Li J, Hu D, Jian J, Chen C, Zheng X and Yang $\mathrm{C}$ : Chemokine (c-x-c motif) ligand 1 is associated with tumor progression and poor prognosis in patients with colorectal cancer. Biosci Rep 38(4), 2018. PMID: 29784873. DOI: 10.1042/BSR20180580

15 Guo F, Zhang J, Wang L, Zhao W, Yu J, Zheng S and Wang J: Identification of differentially expressed inflammatory factors in wilms tumors and their association with patient outcomes. Oncol Lett 14(1): 687-694, 2017. PMID: 28693222. DOI: 10.3892/ ol.2017.6261

16 Ding J, Xu K, Zhang J, Lin B, Wang Y, Yin S, Xie H, Zhou L and Zheng S: Overexpression of cxcl2 inhibits cell proliferation and promotes apoptosis in hepatocellular carcinoma. BMB Rep 51(12): 630-635, 2018. PMID: 30293547. DOI: 10.5483/ BMBRep.2018.51.12.140

17 Xin H, Cao Y, Shao ML, Zhang W, Zhang CB, Wang JT, Liang LC, Shao WW, Qi YL, Li Y, Zhang ZY, Yang Z, Sun YH, Zhang PX, Jia LL and Wang WQ: Chemokine cxcl3 mediates prostate cancer cells proliferation, migration and gene expression changes in an autocrine/paracrine fashion. Int Urol Nephrol 50(5): 861-868, 2018. PMID: 29524043. DOI: 10.1007/s11255018-1818-9 